УДК 537.874 .6

\title{
РАСПОЗНАВАНИЕ ПРОВОДЯЩИХ ОБЪЕКТОВ ПО ХАРАКТЕРИСТИКАМ ОТРАЖЕННОЙ ЭЛЕКТРОМАГНИТНОЙ ВОЛНЫ
}

\author{
ЛЯСОТА Д. В., МОРОЗОВ В. М., МАГРО В. И.
}

Днепропетровский начиональный университет им. О.Т. Гончара, Украина, Днепропетровск, 49050, ул. Научная, 13

\begin{abstract}
Аннотация. Задача дифракции электромагнитной волны на металлических телах решена методом интегрального уравнения. Построены диаграммы обратного рассеивания для четырех различных объектов. На основании вектора признаков, построенного с помощью применения вейвлет-пакетного разложения сигнала, обучена нейронная сеть. Произведено тестирование способности нейронной сети к распознаванию объекта в зависимости от уровня шума. Рассмотрены различные способы формирования вектора признаков.
\end{abstract}

Ключевые слова: дифракция электромагнитной волны; метод интегрального уравнения; вероятностная нейронная сеть; вейвлет пакетное преобразование; распознавание объекта

\section{ВВЕДЕНИЕ}

В настоящее время актуальными являются обратные задачи дифракции, в которых производится определение структуры облучаемого тела на основании параметров отраженной волны [1-3]. Подобные задачи возникают в медицине, биологии, дефектоскопии, радиолокации, геологии и пр. В большинстве случаев задачей является определение наличия того или иного объекта и его распознавание $[2,3]$.

Все более широкое применение в задачах распознавания получают нейронные сети. Их достоинством является способность к обучению и самостоятельному определению характерных признаков, определяющих данный объект. Кроме того, нейронные сети способны в некоторой степени подавлять шум и производить идентификацию объекта на основании его зашумленных характеристик [4]. Однако, при этом необходимо наличие значи- тельного числа элементов в обучающей сеть выборке. Поскольку результаты реального физического эксперимента всегда содержат шум, а иногда являются невозможными или дорогостоящими, в данной работе нейронная сеть обучена на основании теоретически рассчитанных характеристик обратного рассеивания объекта.

Отдельным вопросом является построение вектора признаков, который подается на вход нейронной сети при ее обучении и применении. Построение по определенному алгоритму данного вектора позволяет снизить уровень шума и снижает размерность эталонных векторов признаков, что упрощает их хранение и обработку в системах автоматического распознавания. В данной работе построение вектора признаков производится с помощью адаптивного вейвлет-пакетного разложения (АВПР) сигнала [5]. 